TIT/HEP-204

STUPP-92-131

August, 1992

\title{
Correlation Functions in Two-Dimensional Dilaton Gravity
}

\author{
Yoichiro Matsumura, Norisuke Sakai \\ Department of Physics, Tokyo Institute of Technology \\ Oh-okayama, Meguro, Tokyo 152, Japan \\ YOSHIAKI TANII \\ Physics Department, Saitama University \\ Urawa, Saitama 338, Japan \\ and \\ TAKU UCHINO \\ Department of Physics, Tokyo Institute of Technology \\ Oh-okayama, Meguro, Tokyo 152, Japan
}

\begin{abstract}
The Liouville approach is applied to the quantum treatment of the dilaton gravity in two dimensions. The physical states are obtained from the BRST cohomology and correlation functions are computed up to three-point functions. For the $N=0$ case (i.e., without matter), the cosmological term operator is found to have the discrete momentum that plays a special role in the $c=1$ Liouville gravity. The correlation functions for arbitrary numbers of operators are found in the $N=0$ case, and are nonvanishing only for specific "chirality" configurations.
\end{abstract}


The two-dimensional gravity interacting with a dilaton field and matter fields has attracted much attention recently ever since the work on the black hole evaporation [1]. Many efforts are devoted to study the Hawking radiation and the black hole evaporation in the model [1]- [4]. Most of the works have eventually employed the semi-classical approximation, which is often blamed to be the possible origin of diseases in this problem. Therefore it is very desirable to have a full quantum treatment of the dilaton gravity even for a restricted class of models. In two dimensions, we can perhaps hope to understand quantum theory of the dilaton gravity without using the semi-classical approximations. The continuum approach of the Liouville theory is most suitable in discussing the dilaton gravity, since the theory is nonlinear and it is difficult to invent the discretized version of the model such as matrix models.

The purpose of our paper is to determine the physical states and the gauge invariant operators by a BRST analysis and compute their correlation functions in the dilaton gravity coupled to $N$ massless free scalar fields applying the methods used in the Liouville theory. We shall take the recently proposed models of dilaton gravity [4] that are conformally invariant.

We obtain the BRST cohomology for the case $N \geq 24$ that we cannot find in the existing literature. Apart from the usual string states with momentum and oscillator excitations, we find that there are only a few physical states with nontrivial ghost numbers. This situation is very similar to the case of $0<N<24$ analyzed in ref. [5], but is in sharp contrast to the case of $N=0$ which is essentially the same system as the $c=1$ two-dimensional gravity, except that one of the field is of negative metric. Similarly to ref. [4], we determine the cosmological term by demanding that it should be a gauge (BRST) invariant operator which reduces to the classical cosmological term in the weak coupling limit. The cosmological term in the case of $N=0$ is particularly interesting. We find that the momentum of the cosmological term is precisely at the smallest of the discrete momenta where the characteristic symmetry structure of the $c=1$ two-dimensional gravity has been observed as the ground ring 6.

By applying the methods used in the Liouville theory [7], [8] we compute the correlation functions of gauge (BRST) invariant operators with momentum. The correlation functions up to three gauge invariant operators can be obtained for the general $N>0$ case, and exhibit singularity structures expected from the physical state spectrum. For the $N=0$ case (without matter fields), we obtain correlation 
functions for arbitrary numbers of gauge invariant operators and find that the correlation functions have a singular factor which can be absorbed as a renormalization of the cosmological constant. Since the cosmological term has nonvanishing momentum with positive "chirality," we obtain an interesting selection rule with respect to chirality. The correlation functions are nonvanishing in the case where only one of the gauge invariant operators has negative chirality and all the other operators have positive chirality. All other chirality configurations give vanishing correlation functions.

Let us explain the relevance of the correlation functions to the quantum treatment of the dilaton gravity on the two-dimensional spacetime, such as the black hole evaporation. An insertion of these gauge invariant operators corresponds to creating a hole with appropriate boundary conditions in the language of the physics on the two-dimensional spacetime. If our analysis is extended to macroscopic loops from the local operators, we can find quantum transitions leading to topology change in the two-dimensional dilaton gravity. In order to discuss the black hole evaporation, we need to prepare the appropriate wave function for the black hole and to examine its evolution. We shall use the momentum eigenstates as the gauge invariant operators to compute correlation functions. From the two-dimensional physics point of view, the momentum is just a conserved charge associated with the one dimensional "universe."

First we consider a two-dimensional manifold with a metric $\bar{g}_{\mu \nu}$ whose signature is Euclidean. The Einstein action is a topological invariant in two dimensions. However, it acquires a dynamical meaning when multiplied by a function of a scalar field $\phi$ which we parametrize as $\mathrm{e}^{-2 \phi}$

$$
\begin{aligned}
S_{\text {classical }} & =S_{\text {dilaton }}+S_{\text {matter }}, \quad S_{\text {dilaton }}=\frac{1}{2 \pi} \int d^{2} z \sqrt{\bar{g}}\left[-\mathrm{e}^{-2 \phi} \bar{R}+2 \mu\right], \\
S_{\text {matter }} & =\frac{1}{8 \pi} \int d^{2} z \sqrt{\bar{g}} \sum_{j=1}^{N} \bar{g}^{\mu \nu} \partial_{\mu} f^{j} \partial_{\nu} f^{j},
\end{aligned}
$$

where the scalar field $\phi$ is called dilaton and $N$ free massless scalar fields $f^{j}$ are chosen as matter fields. A function of scalar fields multiplying the Einstein action can be absorbed into the metric by a local Weyl transformation in spacetime dimensions other than two. Therefore the dilaton has a special status in two dimensions: it cannot be eliminated by local Weyl transformations and the Einstein action without the dilaton field is a topological invariant that is dynamically empty. To clarify the significance of the dilaton field more clearly, we can make a local Weyl transformation 
$g_{\mu \nu}=\mathrm{e}^{2 \phi} \bar{g}_{\mu \nu}$

$$
S_{\text {dilaton }}=\frac{1}{2 \pi} \int d^{2} z \sqrt{g} \mathrm{e}^{-2 \phi}\left[-R-4 g^{\mu \nu} \partial_{\mu} \phi \partial_{\nu} \phi+2 \mu\right]
$$

This form of the dilaton gravity system is suggested by the string theory and has been extensively discussed in connection with the black hole evaporation [四]- [4]. In the same spirit, we shall also regard this metric $g_{\mu \nu}$ as physical.

Recently an approach using the conformal field theory was proposed to discuss the quantization of the dilaton gravity [4]. We shall take this approach and give a slight generalization of their models. We can borrow the idea of string theories to quantize the dilaton gravity theory in two dimensions. In particular, the partition function $Z$ is given as a sum over various topologies, and the weight is given by powers of the topological expansion parameter $g_{\mathrm{st}}$ (string expansion parameter), in accordance with the idea of string theories. The contribution $Z_{h}$ from the Riemann surface with $h$ handles is given by a path integral

$$
Z=\sum_{h=0}^{\infty} g_{\mathrm{st}}^{2 h-2} Z_{h}, \quad Z_{h}=\int \frac{\mathcal{D}_{g} g_{\mu \nu} \mathcal{D}_{g} \phi \mathcal{D}_{g} f}{V_{\text {gauge }}} \mathrm{e}^{-S_{\text {classical }}[g, \phi, f]}
$$

where $V_{\text {gauge }}$ is the volume of the group of diffeomorphisms. We use the conformal gauge $g_{\mu \nu}=\mathrm{e}^{2 \rho} \hat{g}_{\mu \nu}$ with the Liouville field $\rho$ and the reference metric $\hat{g}_{\mu \nu}(\tau)$ that depends on the moduli parameters $\tau$ of the Riemann surface. The functional integral over the metric can be converted into an integral over the ghosts $c^{\mu}, b_{\mu \nu}$, the Liouville field and the moduli, which is divided by the volume $V_{\mathrm{CKV}}$ of the group generated by conformal Killing vectors [9].

We have used the physical metric $g_{\mu \nu}$ to define the functional measure in quantizing the matter and the ghost. It is convenient to transform the measure into a measure using $\hat{g}_{\mu \nu}$. The Jacobian is given by the Weyl anomaly which is expressed by the Liouville action $S_{\mathrm{L}}$ [9]

$$
\begin{gathered}
\mathcal{D}_{\mathrm{e}^{2 \rho} \hat{g}} f \mathcal{D}_{\mathrm{e}^{2 \rho} \hat{g}} b \mathcal{D}_{\mathrm{e}^{2 \rho} \hat{g}} c=\mathcal{D}_{\hat{g}} f \mathcal{D}_{\hat{g}} b \mathcal{D}_{\hat{g}} c \mathrm{e}^{-S_{\text {anomaly }}^{\text {gh, },}} \\
S_{\text {anomaly }}^{\text {gh, } f}=\frac{26-N}{12} S_{\mathrm{L}}[\rho, \hat{g}]=\frac{26-N}{24 \pi} \int d^{2} z \sqrt{\hat{g}}\left(\hat{g}^{\mu \nu} \partial_{\mu} \rho \partial_{\nu} \rho+\hat{R} \rho\right) .
\end{gathered}
$$

Since the measure for the Liouville field is nonlinear, the Jacobian for the transformation to the translation invariant measure using $\hat{g}_{\mu \nu}$ is more difficult to determine. 
It has been argued that this Jacobian must be a local functional of the Liouville field $\rho$ containing a bilinear kinetic term, a term linear in the curvature and $\rho$, and the exponential term representing the cosmological term [10]. This is an extremely successful Ansatz. The quantization of the dilaton is similar but may be more complicated. There has also been a proposal for the use of metric $\mathrm{e}^{-2 \phi} g_{\mu \nu}$ rather than $g_{\mu \nu}$ in defining the ghost measure [3]. Therefore, along the spirit of ref. [10], we take the following more general Ansatz for the Jacobian for the transformation of the functional measure to the translationally invariant measure using $\hat{g}_{\mu \nu}$ in the case of the dilaton gravity with the matter

$$
\begin{aligned}
S= & S_{\text {kin }}+S_{\text {cosm }}, \quad S_{\text {kin }}=S_{\text {dilaton }}(\mu=0)+S_{\text {anomaly }}+S_{\text {matter }} \\
S_{\text {kin }}= & \frac{1}{2 \pi} \int d^{2} z \sqrt{\hat{g}}\left[\mathrm{e}^{-2 \phi}\left(-4 \hat{g}^{\mu \nu} \partial_{\mu} \phi \partial_{\nu} \phi+4 \hat{g}^{\mu \nu} \partial_{\mu} \phi \partial_{\nu} \rho-\hat{R}\right)\right. \\
& -\kappa\left(\hat{g}^{\mu \nu} \partial_{\mu} \rho \partial_{\nu} \rho+\hat{R} \rho\right)+a\left(2 \hat{g}^{\mu \nu} \partial_{\mu} \phi \partial_{\nu} \rho+\hat{R} \phi\right) \\
& \left.+b \hat{g}^{\mu \nu} \partial_{\mu} \phi \partial_{\nu} \phi+\frac{1}{4} \sum_{j=1}^{N} \hat{g}^{\mu \nu} \partial_{\mu} f^{j} \partial_{\nu} f^{j}\right]
\end{aligned}
$$

This is the most general Ansatz for the anomaly under the assumption that the measures are defined by $\mathrm{e}^{\alpha \phi} g_{\mu \nu}$ for the quantization of various fields 7 . This is a slight generalization of the Ansatz in refs. [3], [4]. The cosmological term $S_{\text {cosm }}$ will be determined after we discuss the physical states.

We can reduce the above kinetic term to a free field action by a change of variables. In the case of $\kappa \neq 0$, the change of variables reads

$$
\begin{gathered}
\omega=\mathrm{e}^{-\phi}, \quad \chi=-\frac{\rho}{2}-\frac{\omega^{2}+a \ln \omega}{2 \kappa}=\frac{\hat{\chi}}{4 \sqrt{|\kappa|}}, \\
d \Omega=-\frac{1}{\kappa} d \omega \sqrt{\omega^{2}-\kappa+a+\frac{a^{2}+b \kappa}{4 \omega^{2}}}=\frac{d \hat{\Omega}}{4 \sqrt{|\kappa|}} .
\end{gathered}
$$

One should note that the Liouville field $\rho$ in the original metric is contained only in $\hat{\chi}$. Therefore $\hat{\Omega}$ is just a redefined dilaton field and transforms as a genuine scalar field under general coordinate transformations, whereas the field $\hat{\chi}$ transforms as the Liouville field (conformal factor of the metric). This change of variables (6) gives a

${ }^{*}$ If the amount of the anomaly $\gamma_{j}$ is defined by $\mathrm{e}^{2 \alpha_{j} \phi} g_{\mu \nu}$, the parameters are given by $a=\sum \gamma_{j} \alpha_{j}$, $b=\sum \gamma_{j} \alpha_{j}^{2}$. If we allow the coefficients of terms linear in $\hat{R}$ to be arbitrary $\left(-\kappa^{\prime} \hat{R} \rho, \kappa^{\prime} \neq \kappa\right.$ and $a^{\prime} \hat{R} \phi, a^{\prime} \neq a$ ), we cannot make the action into a free field form by the change of variables like (6). 
free field action with the source term for $\hat{\chi}$

$$
S_{\text {kin }}=\frac{1}{8 \pi} \int d^{2} z \sqrt{\hat{g}}\left(\mp \hat{g}^{\mu \nu} \partial_{\mu} \hat{\chi} \partial_{\nu} \hat{\chi} \pm 2 \sqrt{|\kappa|} \hat{R} \hat{\chi} \pm \hat{g}^{\mu \nu} \partial_{\mu} \hat{\Omega} \partial_{\nu} \hat{\Omega}+\sum_{j=1}^{N} \hat{g}^{\mu \nu} \partial_{\mu} f^{j} \partial_{\nu} f^{j}\right)
$$

where the upper (lower) signs are for $\kappa>0(\kappa<0)$. The free field theory is almost identical to the usual Liouville theory with matter fields, except that there is one field with negative metric. The transformed Liouville field $\hat{\chi}$ has negative metric in the case of $\kappa>0$, whereas the transformed dilatonic field $\hat{\Omega}$ has negative metric in the case of $\kappa<0$. For $\kappa=0$, the appropriate change of variables is given by

$$
\chi^{ \pm}=Q\left(-\rho-\frac{b}{2 a} \phi-\frac{2 a+b}{4 a} \ln \left|\mathrm{e}^{-2 \phi}+\frac{a}{2}\right|\right) \pm \frac{2}{Q}\left(\mathrm{e}^{-2 \phi}-a \phi\right) .
$$

In this case, $\chi^{+}+\chi^{-}$transforms as the Liouville field, while $\chi^{+}-\chi^{-}$transforms as a scalar field. The free field action reads

$$
\begin{aligned}
S_{\text {kin }}= & \frac{1}{8 \pi} \int d^{2} z \sqrt{\hat{g}}\left(\hat{g}^{\mu \nu} \partial_{\mu} \chi^{+} \partial_{\nu} \chi^{+}-Q \hat{R} \chi^{+}\right. \\
& \left.-\hat{g}^{\mu \nu} \partial_{\mu} \chi^{-} \partial_{\nu} \chi^{-}+Q \hat{R} \chi^{-}+\sum_{j=1}^{N} \hat{g}^{\mu \nu} \partial_{\mu} f^{j} \partial_{\nu} f^{j}\right) .
\end{aligned}
$$

Actions with different values of $Q$ are related by an $\mathrm{O}(1,1)$ transformation in $\chi^{+}, \chi^{-}$ field space and are equivalent. Finally the parameter $\kappa$ is determined by requiring the conformal invariance (independence on $\hat{g}_{\mu \nu}$ ) of the quantum theory (7) or (9) [4]

$$
\kappa=\frac{N-24}{12}
$$

Let us note that the translation invariant path integral measure for these free fields defines the quantization of the dilaton gravity. In terms of the original variables, the path integral measure may be quite nonlinear, and the path integral region (values of the fields) may sometimes be peculiar. Our attitude is that the dilaton gravity is nonlinear in the original variable and is difficult to quantize. Therefore we can use the free field representation of the kinetic term (7) or (9) together with the translation invariant measure as the definition of (the kinetic term of) the quantum theory of the dilaton gravity.

\footnotetext{
†The eq.(8) has the $a \rightarrow 0$ limit and is valid for $a=0$ too.
} 
To discuss the physical states and the gauge invariant operators of the theory, we compute the cohomology of the BRST charge $Q_{\mathrm{B}}$ for the vanishing cosmological constant $\mu=0$. For the case of $0 \leq N<24$, the analysis of refs. [11], [5] applies with $c=N+1$. Since we have not found the results for $N \geq 24$ in the existing literature, we compute the BRST cohomology for $N \geq 24$ as a direct extension of other cases [11], [5], and find a result similar to the case $0<N<24$ [5]. In the case of $N \neq 24$ it is convenient to introduce the following notation for momentum

$$
p_{I} \equiv\left(-i \beta_{\chi},-i \beta_{\Omega}, p_{j}\right)
$$

corresponding to $X^{I}=\left(\hat{\chi}, \hat{\Omega}, f^{j}\right)$. To raise or lower the indices we should use a metric in field space $\eta_{I J}=(-\kappa /|\kappa|, \kappa /|\kappa|,+1, \cdots,+1)$. For $N=24$, we use $p_{I} \equiv\left(-i \beta_{+},-i \beta_{-}, p_{j}\right)$ and $\eta_{I J}=(+1,-1,+1, \cdots,+1)$.

Let us first describe the chiral (open string) cohomology states for $N>24$. The absolute cohomology consists of $Q_{\mathrm{B}}$ invariant states modulo $Q_{\mathrm{B}}|\Lambda\rangle$, where $|\Lambda\rangle$ is an arbitrary state. The relative cohomology consists of $Q_{\mathrm{B}}$ invariant states annihilated by the antighost zero mode $b_{0}$ modulo $Q_{\mathrm{B}}|\Lambda\rangle$ with $|\Lambda\rangle$ annihilated by $b_{0}$. Usually the absolute cohomology states can be obtained from the relative ones, so we will first obtain the relative cohomology. For $\left(p_{\Omega}, p_{j}\right) \neq 0$, the relative cohomology consists of only the usual string excitations with vanishing ghost number. Therefore the multiplicity of the states with the ghost number $n$ is given by

$$
\operatorname{dim} H_{r e l}^{n}=\left\{\begin{array}{cl}
\delta_{n, 0} P_{N}(R) & \text { for non-negative integer } R \\
0 & \text { otherwise }
\end{array}\right.
$$

where the generating function of $P_{N}(R)$ is the usual partition function for the oscillator excited states at the level $R$ and momentum $p_{I}$ has to satisfy the on-shell condition

$$
\prod_{m=1}^{\infty}\left(1-q^{m}\right)^{-N}=\sum_{R=0}^{\infty} q^{R} P_{N}(R), \quad \frac{1}{2} p^{I} p^{J} \eta_{I J}-i \sqrt{\kappa} p_{\chi}+R=1 .
$$

Even if $\left(p_{\Omega}, p_{j}\right)=0$, the result is the same as eq. (12) unless $p_{\chi}=0$ or $-2 i \sqrt{\kappa}$. Nontrivial cohomology similar to the discrete states in the $c=1$ gravity appear only for the following few cases:

\footnotetext{
$\ddagger$ If we apply a naive hermiticity argument, we find that the momentum $p_{\chi}=-i \beta_{\chi}$ for the field $\hat{\chi}$ should be purely imaginary.
} 
1. For the $p_{I}=0$, we have $N+1$ states with vanishing ghost number and one state with ghost number -1

$$
\alpha_{-1}^{\Omega}\left|p_{I}=0\right\rangle, \quad \alpha_{-1}^{j}\left|p_{I}=0\right\rangle, \quad b_{-1}\left|p_{I}=0\right\rangle,
$$

where $\left|p_{I}\right\rangle$ is the state with momentum $p_{I}$ annihilated by all positive mode oscillators as well as $b_{0}$.

2. For $p_{I} \equiv\left(p_{\chi}, p_{\Omega}, p_{j}\right)=(-2 i \sqrt{\kappa}, 0,0, \cdots, 0)$, we have $N+1$ states with vanishing ghost number and one state with ghost number +1

$$
\alpha_{-1}^{\Omega}\left|p_{I}\right\rangle, \quad \alpha_{-1}^{j}\left|p_{I}\right\rangle, \quad c_{-1}\left|p_{I}\right\rangle
$$

Next we consider the relative cohomology for $N=24$. In this case the result for the generic values of $p_{I}$ is the same as in eqs. (12) and (13) except for the different on-shell condition $-\frac{1}{2} \beta_{+}\left(\beta_{+}+Q\right)+\frac{1}{2} \beta_{-}\left(\beta_{-}-Q\right)+\frac{1}{2} p_{j}^{2}+R=1$. The nontrivial cohomology classes exist only for $p_{I}=0$ or $p_{+}=-p_{-}=i Q, p_{j}=0$

$$
\begin{array}{ccc}
\left(\alpha_{-1}^{+}-\alpha_{-1}^{-}\right)\left|p_{I}=0\right\rangle, & \alpha_{-1}^{j}\left|p_{I}=0\right\rangle, & b_{-1}\left|p_{I}=0\right\rangle \\
\left(\alpha_{-1}^{+}+\alpha_{-1}^{-}\right)\left|p_{+}=i Q\right\rangle, & \alpha_{-1}^{j}\left|p_{+}=i Q\right\rangle, & c_{-1}\left|p_{+}=i Q\right\rangle .
\end{array}
$$

In the present case it can be shown that all states in the relative cohomology are non-trivial states of the absolute cohomology. The remaining states in the absolute cohomology are obtained by multiplying them by the ghost zero mode $c_{0}$ and by adding certain terms if necessary [11]. Namely, we find

$$
H_{a b s}^{n} \simeq H_{r e l}^{n} \oplus c_{0} H_{r e l}^{n-1}
$$

We also obtain the closed string cohomology in the same way as in ref. [12]. The relative cohomology consists of $Q_{\mathrm{B}}+\bar{Q}_{\mathrm{B}}$ invariant states modulo $\left(Q_{\mathrm{B}}+\bar{Q}_{\mathrm{B}}\right)|\Lambda\rangle$, where $|\Lambda\rangle$ is an arbitrary state. The relative cohomology consists of $Q_{\mathrm{B}}+\bar{Q}_{\mathrm{B}}$ invariant states annihilated by the antighost zero modes $b_{0}, \bar{b}_{0}$ modulo $\left(Q_{\mathrm{B}}+\bar{Q}_{\mathrm{B}}\right)|\Lambda\rangle$ with $|\Lambda\rangle$ annihilated by $b_{0}, \bar{b}_{0}$. States in the closed string absolute (relative) cohomology $\mathcal{H}_{\text {abs }}^{n}\left(\mathcal{H}_{\text {rel }}^{n}\right)$ are obtained by taking tensor products of two chiral absolute (relative) cohomology states as left movers and right movers. An important concept in the 
closed string cohomology is the semi-relative cohomology, which consists of $Q_{\mathrm{B}}+\bar{Q}_{\mathrm{B}}$ invariant states annihilated by $b_{0}-\bar{b}_{0}$ modulo $\left(Q_{\mathrm{B}}+\bar{Q}_{\mathrm{B}}\right)|\Lambda\rangle$ with $|\Lambda\rangle$ annihilated by $b_{0}-\bar{b}_{0}[12$. In the present case it can be shown that

$$
\mathcal{H}_{\text {semi }}^{n} \simeq \mathcal{H}_{\text {rel }}^{n} \oplus\left(c_{0}+\bar{c}_{0}\right) \mathcal{H}_{\text {rel }}^{n-1}
$$

The cosmological term is chosen from these BRST cohomology classes by imposing the additional requirement [4] that it should be of the same form as the original cosmological term in the limit of weak gravitational coupling $\mathrm{e}^{\phi} \rightarrow 0$. We obtain uniquely

$$
S_{\text {cosm }}= \begin{cases}\frac{\mu}{\pi} \int d^{2} z \sqrt{\hat{g}} \mathrm{e}^{\frac{1}{\sqrt{|\kappa|}}(-\hat{\chi}+\hat{\Omega})} & \text { for } \kappa \neq 0, \\ \frac{\mu}{\pi} \int d^{2} z \sqrt{\hat{g}} \mathrm{e}^{-\frac{1}{Q}\left(\chi^{+}+\chi^{-}\right)} & \text {for } \kappa=0 .\end{cases}
$$

The particularly interesting is the case of no additional matter fields, i.e., $N=0$ $(\kappa=-2)$. By identifying $\kappa=-2$ in eq. (7), we find that the dilaton gravity system without additional matter corresponds to the case of the Liouville gravity coupled to the $c=1$ conformal matter. The only distinction is that the $c=1$ matter comes from the dilaton degree of freedom, and that the resulting free boson $\hat{\Omega}$ has negative metric. To compare with the physical operators in the Liouville gravity, we should rotate the free boson to purely imaginary values $\hat{\Omega}=i \bar{\Omega}$ and identify it with the usual free boson with the positive metric. Then we find that the two-momentum of this cosmological term is precisely the simplest discrete value [6] $\left(\beta_{\chi}, p_{\bar{\Omega}}\right)=\left(-i p_{\chi}, i p_{\Omega}\right)=(-1 / \sqrt{2}, 1 / \sqrt{2})$.

Among these BRST cohomology classes, we shall take momentum eigenstates (gravitationally dressed tachyon vertex operators in the language of the Liouville gravity), and compute their correlation functions. In the case of $N \neq 24$

$$
\begin{gathered}
O_{p}=\int d^{2} z \sqrt{\hat{g}} \mathrm{e}^{\beta_{\chi} \hat{\chi}+\beta_{\Omega} \hat{\Omega}+i p_{j} f^{j}} \\
-\frac{1}{2} \beta_{\chi}\left(\beta^{\chi}+2 \sqrt{|\kappa|}\right)-\frac{1}{2} \beta_{\Omega} \beta^{\Omega}+\frac{1}{2} p_{j} p^{j}=1 .
\end{gathered}
$$

The $(N+2)$-momentum of the cosmological term is $q_{I}=(i / \sqrt{|\kappa|},-i / \sqrt{|\kappa|}, 0, \cdots, 0)$ and that of the background source is $Q^{I}=(-2 i \sqrt{|\kappa|}, 0,0, \cdots, 0)$.

\footnotetext{
${ }^{\S}$ We have fixed the integration constant in the field transformation (6) between $\omega$ and $\Omega$ by the asymptotic form $\Omega \rightarrow-\left(\omega^{2}+(a-\kappa) \ln \omega\right) /(2 \kappa)+O\left(1 / \omega^{2}\right)$.
} 
It has been noted that the integration over the zero mode of the Liouville field has to be done without using perturbation theory in terms of the cosmological constant [7], [8]. By the same token, we perform the integration over the zero mode of the transformed Liouville field $\hat{\chi}$ without expanding in powers of the cosmological constant in the action. After integrating over the zero mode of $\hat{\chi}$ along the real axis, we integrate over the zero mode of $\hat{\Omega}$ along the imaginary axis to obtain the momentum conservation delta function II. In this way we find the path integral with cosmological term insertions

$$
\begin{aligned}
&\left\langle\prod_{k=1}^{n} O_{p_{k}}\right\rangle=\int \frac{[d \tau] \mathcal{D}_{\hat{g}} \hat{\chi} \mathcal{D}_{\hat{g}} \hat{\Omega} \mathcal{D}_{\hat{g}} f}{V_{\mathrm{CKV}}} \mathrm{e}^{-S[\hat{\chi}, \hat{\Omega}, f, \hat{g}]} O_{p_{1}} \cdots O_{p_{n}} \\
&=(2 \pi)^{N+1} \delta^{N}\left(\sum_{k=1}^{n} p_{k}\right) \delta\left(\sum_{k=1}^{n} \beta_{\Omega k}+\frac{s}{\sqrt{|\kappa|}}\right) \sqrt{|\kappa|} \Gamma(-s) \tilde{A}\left(p_{1}, \cdots, p_{n}\right), \\
& \tilde{A}=\int \frac{[d \tau] \prod_{k=1}^{n}\left[d^{2} z_{k} \sqrt{\hat{g}\left(z_{k}\right)}\right]}{V_{\mathrm{CKV}}}\left\langle\prod_{k=1}^{n} \mathrm{e}^{i p_{k} \cdot \tilde{X}\left(z_{k}\right)}\left(\frac{\mu}{\pi} \int d^{2} w \sqrt{\hat{g}} \mathrm{e}^{i q \cdot \tilde{X}}\right)^{s}\right\rangle_{\tilde{X}},
\end{aligned}
$$

where $s$ is the number of cosmological term insertions and the $(N+2)$-momentum conservation reads

$$
s=\sqrt{|\kappa|} \sum_{k=1}^{n} \beta_{\chi k}-2 \kappa(1-h), \quad \sum_{k=1} p_{k}^{I}+s q^{I}=-Q^{I}(1-h) .
$$

The expectation value in eq. (21) denotes the functional integral over the nonzero modes $\tilde{X}^{I}$. As is usual in the Liouville gravity, the zero mode integration is done assuming $s<0$. If we continue analytically (in external momenta and/or the central charge) to a nonnegative integer $s$, we can integrate the non-zero mode functional integral in $\tilde{A}$ by means of the usual free field contractions. Finally we analytically continue the result to desired values of $s$.

For the case of $N \neq 24,0$, we find the three-point correlation functions on the sphere

$$
\tilde{A}\left(p_{1}, p_{2}, p_{3}\right)=\left[\mu \Delta\left(1+p_{1} \cdot q\right) \Delta\left(1+p_{2} \cdot q\right) \Delta\left(1+p_{3} \cdot q\right)\right]^{s},
$$

where $\Delta(x) \equiv \Gamma(x) / \Gamma(1-x)$. We see that the three-point functions exhibit singularity structures corresponding to the physical states obtained in the BRST analysis.

${ }^{\top}$ An arbitrary linear combination of two variables $\hat{\chi}$ and $\hat{\Omega}$ can be used instead of $\hat{\chi}$ and its orthogonal combination instead of $\hat{\Omega}$ to give the same result, provided the latter variable is integrated along the imaginary axis. 
However, the peculiarity of the correlation functions is that these singularities are all raised to a power of the cosmological term insertions $s$. It is not difficult to see the origin of these singularities from the operator product expansion. The cosmological term has the null momentum $q_{I}$ and the integration over the positions of the cosmological term operators is factorized. The singularities are due to the short distance singularities between these cosmological term operators and the inserted gauge invariant operators. We can obtain two-point function from the three-point function if we put one of the momenta to that of the cosmological term $q_{I}$, replace $s$ by $s-1$, multiply by $-1 / \pi$, and integrate in $\mu$ once. Similarly, the one-point function is obtained by repeating the same procedure for the two-point function. We find that these correlation functions vanish for $s \in \mathbf{Z}, s>0$ because $1+q \cdot q=1$. Therefore the analytic continuation gives vanishing two- and one-point functions.

For the $N=24$ case we obtain the three-point correlation functions on the sphere

$$
\left\langle\prod_{k=1}^{3} O_{p_{k}}\right\rangle=(2 \pi)^{25} \delta^{24}\left(\sum_{k=1}^{3} p_{k}\right) \delta\left(\frac{1}{Q} \sum_{k=1}^{3}\left(\beta_{+k}-\beta_{-k}\right)+2\right) \Gamma(-s) \tilde{A}\left(p_{1}, p_{2}, p_{3}\right)
$$

with the same amplitude $\tilde{A}$ in eq. (23) and the number of cosmological term insertion $s$ is given by

$$
s=\frac{Q}{2} \sum_{k=1}^{3}\left(\beta_{+k}+\beta_{-k}\right) .
$$

For $N=24$ the momenta of the cosmological term and the background source are $q_{I}=\left(\frac{i}{Q}, \frac{i}{Q}, 0, \cdots, 0\right)$ and $Q_{I}=(-i Q, i Q, 0, \cdots, 0)$ respectively, and momentum conservation holds in the same form as eq. (22). Since $q \cdot q=0$ is still valid, one and two point functions vanish in this case too.

In the case of $N=0$, we can obtain correlation functions for arbitrary numbers $n$ of gauge invariant operators $(20)$ on the sphere. Since the cosmological term in this case is at the special discrete momentum, the correlation functions become singular due to their insertions [8]. Therefore we need to keep the momentum $q_{I}$ of the cosmological term at a generic value to regularize the correlation functions. After an analytic continuation in $s$ analogous to the $c=1$ Liouville gravity theory, we obtain the $n$-point correlation functions. In the case of $N=0$, it is important to distinguish two solutions of the on-shell condition (20)

$$
\beta_{\chi}^{( \pm)}=-\sqrt{2} \pm \beta_{\Omega}
$$


Following the terminology of Liouville gravity [8], we call the solution with the + $(-)$ sign as positive (negative) chirality. We find the correlation functions of $n$ gauge invariant operators with chiralities $(-,+, \cdots,+)$

$$
\begin{aligned}
\left\langle\prod_{k=1}^{n} O_{p_{k}}\right\rangle= & 2 \pi \delta\left(\frac{1}{\sqrt{2}} \sum_{k=1}^{n}\left(\beta_{\chi k}+\beta_{\Omega k}\right)+2\right) \Gamma(-s) \\
& \times[\mu \Delta(-\rho)]^{s} \frac{\pi^{n-3}}{\Gamma(n+s-2)} \prod_{k=2}^{n} \Delta\left(1-\sqrt{2} \beta_{\Omega k}\right),
\end{aligned}
$$

where the regularization parameter $\rho=q \cdot q / 2$ should be set to zero eventually. The correlation functions are very similar to the Liouville gravity case, but are singular due to the discrete momentum for the cosmological term. We can absorb the divergence as a renormalization of the cosmological constant

$$
\mu_{r}=\mu \Delta(-\rho)
$$

Correlation functions vanish identically for chirality configurations other than the above one. When computing these correlation functions, one should keep the regularization parameter $\rho$ to be nonvanishing in order to avoid ambiguous results.

We hope that the above results on the physical states and the correlation functions may serve as a first step for a full quantum treatment of the dilaton gravity beyond the usual semi-classical approximations. Let us emphasize that the continuum approach is flexible enough to study the dilaton gravity, whereas the powerful method of matrix models is yet to be applied to this case. However, when we are writing this paper, we received an interesting preprint which computed the partition function with the tachyon background for the $c=1$ quantum gravity by using matrix model approach [13]. We are currently studying to apply their results to our case of $N=0$ dilaton gravity. The wave functions of the universe and possible consequences on the black hole evaporation are being studied.

We thank T. Mishima, A. Hosoya, and A. Nakamichi for a useful discussion and for informing us their results prior to publication. One of us (N.S.) thanks H. Kawai and C. G. Callan for a discussion. This work is supported in part by Grant-in-Aide for Scientific Research for Priority Areas from the Ministry of Education, Science and Culture (No. 04245211). 


\section{References}

[1] C.G. Callan, S.B. Giddings, J. Harvey and A. Strominger, Phys. Rev. D45 (1992) R1005.

[2] J. Russo and A.A. Tseytlin, Stanford preprint SU-ITP-92-2 (1992); J. Russo, L. Susskind and L. Thorlacius, Stanford preprint SU-ITP-92-4 (1992); T. Banks, A. Dabholkar, M. Douglas, and M. O'Loughlin, Phys. Rev. D45 (1992) 3607; S.W. Hawking, Phys. Rev. Lett. 69 (1992) 406; D. Cangemi and R. Jackiw, Phys. Rev. Lett. 69 (1992) 233; T.T. Burwick and A.H. Chamseddine, Zürich preprint ZU-TH-4/92 (1992); S.W. Hawking and J.M. Stewart, DAMTP preprint (1992).

[3] A. Strominger, Santa Barbara preprint UCSBTH-92-18 (1992).

[4] A. Bilal and C. Callan, Princeton preprint PUPT-1320 (1992); S.P. de Alwis, Colorado preprints COLO-HEP-280, 284, 288 (1992); S.B. Giddings and A. Strominger, Santa Barbara preprint UCSB-TH-92-28 (1992); A. Miković, Queen Mary preprint QMW/PH/92/12 (1992).

[5] A. Bilal, Phys. Lett. B282 (1992) 309.

[6] E. Witten, Nucl. Phys. B373 (1992) 187; J. Avan and A. Jevicki, Phys. Lett. B266 (1991) 35; D. Minic, J. Polochinski and Z. Yang, Nucl. Phys. B369 (1992) 324; S. Das, A. Dhar, G. Mandal and S. Wadia, Mod. Phys. Lett. A7 (1992) 71, 937; I.R. Klebanov and A.M. Polyakov, Mod. Phys. Lett. A6 (1991) 3273; Y. Matsumura, N. Sakai and Y. Tanii, Tokyo Inst. of Tech. and Saitama preprint TIT/HEP-186, STUPP-92-124 (1992).

[7] M. Goulian and M. Li, Phys. Rev. Lett. 66 (1991) 2051; A. Gupta, S. Trivedi and M. Wise, Nucl. Phys. B340 (1990) 475.

[8] P. Di Francesco and D. Kutasov, Phys. Lett. B261 (1991) 385; Nucl. Phys. B375 (1992) 119; Y. Kitazawa, Phys. Lett. B265 (1991) 262; N. Sakai and Y. Tanii, Prog. Theor. Phys. 86 (1991) 547; V.S. Dotsenko, Mod. Phys. Lett. A6 (1992) 3601.

[9] A.M. Polyakov, Phys. Lett. B103 (1981) 207; D. Friedan, in Recent Advances in Field Theory and Statistical Mechanics, eds. J.B. Zuber and R. Stora, (North-Holland, Amsterdam, 1984). 
[10] F. David, Mod. Phys. Lett. A3 (1988) 1651; J. Distler and H. Kawai, Nucl. Phys. B321 (1989) 509.

[11] B.H. Lian and G.J. Zuckerman, Phys. Lett. B254 (1991) 417; B266 (1991) 21;

P. Bouwknegt, J. McCarthy and K.Pilch, Commun. Math. Phys. 145 (1992) 541; N. Ohta, Osaka preprint OS-GE 26-92 (1992).

[12] E. Witten and B. Zwiebach, Nucl. Phys. B377 (1992) 55.

[13] R. Dijkgraaf, G. Moore and R. Plesser, Princeton preprint IASSNS-HEP92/48 (1992). 